\title{
Teaching Fluids to IPLS Students from a Microscopic Viewpoint
}

\author{
Daniel E. Young and Dawn C. Meredith \\ Department of Physics, University of New Hampshire, 9 Library Way, Durham, NH 03824
}

\begin{abstract}
For introductory life science students, fluid dynamics is a topic of great relevance, yet difficult to understand conceptually. This pilot study focuses on implementing and assessing curricular interventions to help students understand pressure, vacuums, and pressure gradients. Our pedagogical strategy was to focus on mechanistic reasoning and a multiple-scale view of fluids. Student learning was assessed through open-ended conceptual questions administered before and after instruction. Initial results show that significant gains were achieved.
\end{abstract}

Keywords: Fluids, Pressure, Mechanistic Reasoning

PACS: 01.40.-d, 01..40.G-, 51.10.+y, 47.15.-x

\section{INTRODUCTION}

For life science majors, fluid dynamics is a difficult yet essential topic: organisms live in fluid environments and fluids inside organisms fulfill essential roles including structure and transport. Traditionally, fluids receive a cursory treatment in introductory physics courses, but introductory physics for life science majors (IPLS) is often the last physics course for these students, so fluids must be treated as comprehensively as possible [1]. In this paper we describe and assess a curricular intervention for fluid dynamics that combines both a molecular view of matter and mechanistic reasoning.

There is a wealth of data regarding student misconceptions about fluids and microscopic reasoning. Loverude et al. [2] have investigated student understanding of buoyancy and hydrostatics and found a number of difficulties. Notably, they found that students' difficulties with Newton's second law negatively impact their ability to understand hydrostatics. Similarly, Kautz et al. [3] investigated student understanding of the ideal gas law and found that many of the student difficulties that were identified at the macroscopic level seemed to be rooted in incorrect or incomplete microscopic models of gases.

In this paper we begin with a description of the pedagogical strategies we used to design our presentation of fluid dynamics. Then we describe our data collection, focusing on student reasoning about motion of fluid up a straw.

\section{PEDAGOGICAL STRATEGIES AND GOALS}

Developing students' use of mechanistic reasoning was our first pedagogical strategy. Russ et al. [4] describe this kind of reasoning as follows: “...by knowing the general properties of entities and activities, much can be said about what must have produced them at earlier stages and what they can produce in subsequent steps." This form of reasoning is well within students' abilities [5] and allows them to reason in novel situations. This approach pairs well with Newton's second law to give clear explanations of otherwise perplexing phenomena in fluid dynamics.

Based on initial encouraging data [6, 7], we chose to use a multiple scale description of fluids to increase learning gains. The term "multiple scales" refers to visualizing and conceptualizing fluids at multiple length scales simultaneously.

To clarify how mechanistic and multi-scale reasoning can be used to explain fluid flow, we examine the motion of fluid up a straw. Air molecules (microscopic scale) collide with the top layer of liquid (mesoscopic scale) thereby exerting pressure on the fluid layer. When the person inhales, the number of molecules in the straw decreases (decreasing the number of collisions), and therefore decreasing the pressure. This newly created pressure gradient between the mouth and the column of fluid below the straw (macroscopic scale) causes the upward acceleration of the fluid in the straw.

We classify the above explanation as mechanistic (as opposed to merely causal) because "it concerns the process underlying the association" [4], that is, it explains pressure as due to collisions. An explanation 
that includes only a pressure differential without explaining the underlying process would be causal.

We had two reasons to believe that the multi-scale approach would be accessible to IPLS students. First, life science students are asked to think about multiple length scales in their own discipline (molecules, cells, tissues, organs, organisms, and ecology), so we posited that they would be comfortable using similar reasoning in physics. Second, almost all of our students had taken college chemistry previously and had been introduced to the kinetic theory of gases, which is based on a molecular viewpoint.

Mechanistic and multi-scale reasoning were used several times throughout the course to help students become comfortable and familiar with these approaches, and the students worked with these ideas in both conceptual group work and homework. For example, the adjustability of the normal force was explained with a ball and spring model of solids, students reviewed the kinetic theory of gases, and used the PhET [8] fluid flow simulation to help them visualize fluids at the molecular scale. Changing pressure in a horizontal pipe of various widths (i.e., Bernoulli's equation at constant height) was explained using mass conservation and the need for a pressure differential to accelerate a mesoscopic wall of water.

\section{METHODOLOGY}

To assess the effectiveness of this approach, data was collected from 48 students during an accelerated 5-week summer IPLS course at the University of New Hampshire. Forty-one of these students were in life science related majors and all but one had taken college level general chemistry prior to our instruction. Our course covered kinematics, dynamics, fluids, and energy.

Data was gathered from conceptual open-ended questions on pre-assessments, interviews, homework, and exams. In addition, an end-of-class survey asked students to reflect on their use of mechanistic and multi-scale approaches.

The data was then analyzed using an approach informed by grounded theory [9], including open coding and inter-rater reliability. After looking for general patterns within the data, broad categories were identified and we classified student responses based on how well they matched an expert's conceptual understanding.

\section{RESULTS}

In this section, we focus on student understanding of the mechanism for fluid flow up a straw. This mechanism in turn is built on a microscopic understanding of pressure and vaccums, which will be discussed first.

\section{Kinetic Theory of Gases}

We determined the initial level of student understanding of the kinetic theory of gases through a pre-assessment. We first asked about the microscopic distribution of gases: "Say we have a box that contains six (6) molecules of a gas (we will call this “case A"). Using one circle for each molecule, sketch in the box provided how the gas molecules will typically arrange themselves in the chamber." All but three students drew molecules filling the chamber evenly, moving with similar velocities in all directions.

Next they were asked to reason about a container of gas, as various parameters were changed. For example, "Is the pressure in case D (double the volume) going to be greater than, less than, or equal to the pressure in case A? Explain your reasoning based on the sketches you drew without appealing to an equation." Of the forty-five students, only six responded incorrectly about the differences in pressure. Of those who responded correctly, twenty attributed the decrease in pressure to the increased volume without further elaboraion, three gave no reasons, two appealed to equations, and the remaining fourteen attributed the pressure difference to fewer microscopic collisions with the walls.

We concluded that students did have some knowledge of the kinetic theory on which we could build, but that most lacked the full mechanistic reasoning of particulate collisions resulting in pressure on the walls. Rather, some seem to appeal to an equational relation between the two ideal gas law variables of pressure and volume without considering the mechanistic process behind the cause.

On the post-instruction exam regarding fluids, students were asked "You have a setup with a closed bulb filled with air at atmospheric pressure on the left connected to a curved tube containing water that is open to the air on the right. If you use your hands to surround and warm the air in the bulb, sketch what will happen to the fluid level in the tube. Explain your reasoning." Twenty seven of the students responding gave full mechanistic reasoning. For example, one student responded, “Temperature will increase kinetic energy of the gas molecules, resulting in more collisions. This will mean more pressure on the left, and that will push the fluid in the direction of the right side until there is a balance." Twelve responses lacked one piece of the mechanism, and six responses lacked any coherent sense of mechanism. In summary, for the kinetic theory of gasses we see an increase from 31\% to $60 \%$ of students who give full mechanistic reasoning, with an additional $27 \%$ giving partial 
mechanistic reasoning on the post-test, and 14\% giving no mechanistic reasoning.

\section{Vacuum}

As we wished to use the idea of a vacuum to present pressure as a pushing force as opposed to a pulling one, we assessed students' understanding of a vacuum and its properties. On the pre-assessment, students were asked: "Explain the notion of a "vacuum" (not the cleaning variety) using either a sketch similar to the ones drawn previously or by providing a detailed description." Eighteen students responded that a vacuum was nothing or no molecules, ten defined a vacuum as something with no force or no pressure, and eighteen students had incorrect, blank, or nonsensical answers. Student responses to the next question, "What is the pressure reading inside of a vacuum?" were categorized as follows: twenty-five students responded that a vacuum had zero pressure, two said it had a high pressure, and two that it has a negative pressure. None of these students explained that a vacuum had no pressure due to lack of collisions. The remaining seventeen responses were nonsensical or blank

On the post-instruction fluids exam, students were asked: "Explain from a molecular point of view why a vacuum cannot suck or pull on a liquid.” All but seven students reasoned correctly using ideas of fewer molecules (30 students) and/or no pressure or force (27), indicating their evolved use of mechanisms though their reasoning was not always at the level of mechanistic chaining. One example of complete student reasoning using collisions was as follows: "molecules cannot suck or pull anything. They can collide with each other to create a pushing force, not a pulling force."

TABLE 1. Student responses to vacuum questions. For the pre-test, students were included in only one category; for the post-test they were included in multiple categories.

\begin{tabular}{|c|c|c|}
\hline $\mathbf{N = 4 6}$ & Pre-test & Post-test \\
\hline Fewer or no molecules & $39 \%$ & $65 \%$ \\
\hline Lower pressure & $22 \%$ & $59 \%$ \\
\hline Fewer collisions & $0 \%$ & $26 \%$ \\
\hline Blank or incorrect & $39 \%$ & $15 \%$ \\
\hline
\end{tabular}

\section{Motion of Fluid up a Straw}

Lastly, we turn to student understanding of fluid moving up a straw. Similar questions were asked pre and post instruction:

(Pre) "It is well known that you can draw liquid from a glass up a drinking straw and into your mouth. Explain how the liquid is able to move up the straw using your previous sketches or any of the principles discussed as evidence (pressure, temperature, vacuum, etc.)."

(Post) "How does the presence of a vacuum at the top of a straw make the fluid accelerate upwards?"

Codes of student responses are given in Table 2 . While there is persistence in using the explanations of "sucking" and "wanting to be filled" there is a large shift toward the use of pressure gradients and away from unclear or blank answers.

Compared to the responses on the vacuum question, fewer students explained motion up the straw by referencing collisions. We also note that $32 \%$ gave incorrect answers as opposed to $15 \%$ in the vacuum question. We will return to this difference in the conclusion.

TABLE 2. Student responses to the straw problem. For the first four rows, each student is included only once. The last two rows count some students twice.

\begin{tabular}{|c|c|c|}
\hline $\mathbf{N = 4 1}$ & Pre-test & Post-test \\
\hline Unclear or blank & $37 \%$ & $2 \%$ \\
\hline Vacuums want to be filled & $15 \%$ & $10 \%$ \\
\hline Sucking or pulling force & $24 \%$ & $20 \%$ \\
\hline $\begin{array}{c}\text { Lower pressure without } \\
\text { reference to gradient }\end{array}$ & $24 \%$ & $0 \%$ \\
\hline Pressure gradient & $15 \%$ & $69 \%$ \\
\hline Fewer collisions & $2 \%$ & $20 \%$ \\
\hline Blank or incorrect & $76 \%$ & $32 \%$ \\
\hline
\end{tabular}

\section{Student Survey Responses}

Gains in student understanding of target phenomena were key to judging the effectiveness of our curriculum. However, there were several questions that were best answered by asking the students directly on an anonymous survey at the end of class $(\mathrm{N}=35)$. Here we present our rationale for the questions, student responses, and our conclusions.

We asked: "Have you encountered our multi-scale reasoning before in your biology or chemistry classes?" Twenty students said yes and fifteen said no. This indicated that we could not count on familiarity with multi-scale reasoning as we had initially assumed. Therefore, we plan on introducing multi-scale reasoning in the familiar context of time management (with scales from minutes to decades) to help students see the value of such reasoning.

In order to assess student's familiarity with the ideas of fluid flow we asked: "After our fluids instruction do you feel comfortable discussing ideas such as blood flow and how straws work from a physics context?" Twenty-nine students replied that they were comfortable, six (17\%) said they were not; 
these numbers are in rough agreement with student success on the vacuum question. Typical "yes" and "no" responses are given below.

"Yes...the straw example was new to me and now makes more sense. Previously I had understood the concept as vacuum and the nature of gases to expand outward to fill a container. Relating it with the forces of air pressure made [it] clearer."

"No...I thought that the way that blood flow examples were taught was very confusing but I did like seeing how physics relates back to real life scenarios."

We also asked, "What does "mechanistic reasoning' mean to you?" to get a sense if we clearly communicated these ideas to students. Four (12\%) out of the thirty-five students either responded that they did not know, or felt it did not help. The remaining students gave a variety of answers that were reasonably in line with our goals. For instance, one student responded: "For me, mechanistic reasoning means understanding the mechanism behind why something works rather than simply accepting that something as fact. Understanding the mechanism behind something allows you to put the pieces together for yourself, so that even if you forget what the end result is supposed to be, you can work your way through the mechanism to figure it out for yourself."

\section{CONCLUSIONS}

We have developed instructional interventions on fluid dynamics using mechanistic and multi-scale reasoning. We assessed the interventions by comparing student pre and post instructional responses to conceptual questions and the initial results are encouraging. We have seen large gains in students' ability to explain both the mechanism behind both zero pressure in a vacuum and the motion of fluid up a straw. However, we still see about $15 \%$ of our students struggling with understanding moving fluids across different contexts, and this same percentage show dissatisfaction and confusion on the survey. Additionally, results from the survey indicate that the majority of students understand and appreciate our pedagogical approaches.

We return here to the issue mentioned in the section on motion of fluid up a straw. In that question, many students used a pressure differential (instead of fewer collisions at the top) as their explanation. We expect that most physicists would have answered similarly and not referred to collisions. This is an example of an issue discussed by Russ et al. [4]: explanations "bottom out" at different places in different contexts.
This raises two pedagogical questions: do we always want explanations in this course in terms of collisions? And if not, how do we effectively communicate to students what level of explanation is sufficient for a given question? The answer to the second question seems straightforward: we can explicitly ask students to explain at the microscopic level (for collisions) or the macroscopic level (for pressure gradients).

To begin to answer the first question, we return to the difference in success between the vacuum question $(15 \%$ incorrect) and the straw question (32\% incorrect). We speculate that this difference may be that, in the vacuum question we explicitly asked for reasoning at the molecular level, while in the straw question we did not. This raises a question that requires further investigation: are some students are in a "middle space" where thinking at the microscopic level triggers correct reasoning, but thinking at the macroscopic level triggers incorrect reasoning? Are other students successful in either context?

Based on our initial data we are confident in claiming that the majority of students are comfortable reasoning with the multiple scale model, and they do so productively when asked to describe fluidic situations. Our goal now, based on student feedback, is to further enhance the model through development of relevant lecture demonstrations and tutorials. Additionally, we would like to extend this approach to Bernoulli's equation and viscous flow.

\section{ACKNOWLEDGEMENTS}

We thank James Vesenka, Katherine Misaiko and Elizabeth K. Whitmore for many valuable conversations. This research is supported by NSF DUE grant number 1044211.

\section{REFERENCES}

1. Meredith, D. \& Bolker, J. American Journal of Physics 80, 913922 (2012).

2. Loverude, M. E., Kautz, C. H., \& Heron, P. R. L. American Journal of Physics 77, 1178-1187 (2003).

3. Loverude, M. E., Heron, P. R. L., \& Kautz, C. H. American Journal of Physics 78, 75-85 (2009).

4. Russ, R. S., Scherr, R. E., Hammer, D., \& Mikeska, J. Science Education 92.3, 499-525 (2008).

5. Redish, E. F. \& Hammer, D. American Journal of Physics 77, 629- 642 (2009).

6. Robertson, A. D. \& Shaffer, P. S. American Journal of Physics 81, 303-312 (2013).

7. Vesenka, J. private communication.

8. http://PhET.colorado.edu

9. Creswell, J. W. Qualitative Inquiry \& Research Design: Choosing among Five Approaches, $2^{\text {nd }}$ Ed. (Sage Publications, Thousand Oaks, CA, 2007), pp. 62-67. 